ANL-HEP-PR-92-40

June 15, 1992

\title{
LIGHT QUARKS AND SMALL X PHYSICS *
}

\author{
Alan R. White \\ High Energy Physics Division \\ Argonne National Laboratory \\ Argonne, IL 60439
}

\begin{abstract}
The significance of the low $k_{\perp}$ part of the Lipatov equation for the QCD soft Pomeron is discussed. It is then argued that light quarks are essential for the emergence of confinement and a Pomeron with the right physical properties. The implications for small $\mathrm{x}$ parton distributions are considered.
\end{abstract}

\section{MASTER}

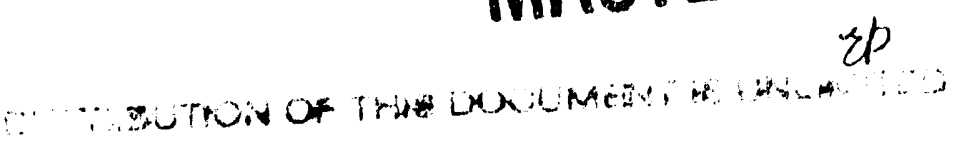
\footnotetext{
"Work supported by the U.S. Department of Energy, Livision of High Energy Physics, Contract
W-31-109-ENG-38.
} 


\section{INTRODUCTION}

Theoretical discussion of the small $x$ behavior of parton distributions is currently dominated by questions of the applicability of the Lipatov equation[1], the meaning of the perturbative Pomeron etc.[2]. The Lipatov equation is satisfied by a subset of the general set of reggeon diagrams that describe the Regge limit in gauge theories. In this talk I will begin by discussing the signifcance of the low $k_{\perp}$ part of the Lipatov equation, and of general reggeon diagrams, for the so-called "soft Pomeron". I will then argue that light quarks are essential for understanding how confinement works in the Regge limit and bow a (soft) Pomeron with the right physical properties can emerge from the low $k_{\perp}$ region of reggeon diagrams. I shall try to give qualitative arguments that can be followed without an intricate knowledge of such diagrams. Some of the basic elements of my work on the Pomeron[3] will be involved - although not the whole story! Finally I will discuss the implications for studies of small $x$ parton distributions - including the phenomenological issue of the separation of perturbative, or "hard Pomeron" effects from "non-perturbative" soft Pomeron effects.

\section{THE LIPATOV EQUATION AND MULTI-REGGE THEORY}

The "Lipatov equation" goes back to the pioneering work' of Fadin, Kuraev and Lipatov[4], and Cheng and Lo[5] on the Regge limit of massive (spontaneously broken) Yang-Mills theories.

-Work supported by the U.S. Department of Energy, Division of High Energy Physics, Contract W-31-109ENG-38.
The build-up of Regge behavior in such theories is dominated by "on-shell" regions of Feynman diagrams. This is particularly clear when dispersion theory techniques are used $[1,3,6]$, and we can illustrate it as follows. First we consider the sum of all tree diagrams for an elastic scattering amplitude. In the Regge limit this gives

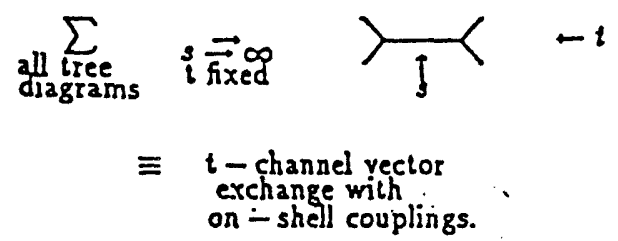

We consider next the multi-Regge limit for the sum of all tree diagrams for a general production amplitude. This is given by on-shell vector exchange in each t-channel. When such production amplitudes are inserted into the (s-channel) unitarity equation (and a dispersion relation utilized) the leading log result is the reggeization of elastic scattering vector exchange, that is

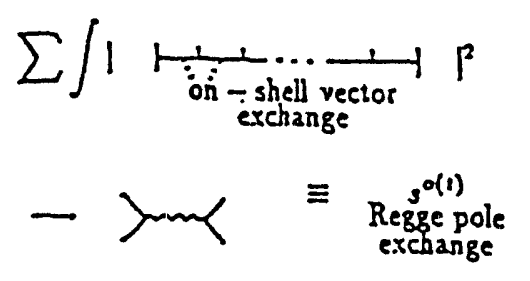

Similarly the multi-Regge leading-log result for production amplitudes (computed from multiple 
discontinuity equations $[3,6])$ is the reggeization of all vector exchanges. If the reggeized production amplitudes are inserted in the unitarity equation then (at the leading-log level) we find that Regge pole behavior is self-consistently "bootstrapped". The next-to-leading level gives (after Fourier transforming from Ins to complex angular momentum) the massive form of what is now referred to as the Lipatov equation, that is in terms of reggeon diagrams

$$
\begin{aligned}
& \sum \int 1 \text { Rerǵte pole exchange } 1^{2} \\
& \rightarrow \text { bind } \equiv \operatorname{sum}_{\substack{\text { suggeon } \\
\text { diagrams } \\
\text { diagrams }}} \\
& \lim =\gamma \sim \alpha+\gamma= \\
& \equiv \text { Lipatov equation }
\end{aligned}
$$

This process can be continued $[3,6,7]$ to construct (in principle) the complete set of reggeon diagrams. For our purposes it will be sufficient to note that such diagrams contain a reggeon propagator (an "energy denominator" with angular momentum playing the role of energy) for each reggeon state, interactions which are functions of transverse momenta, and integrals over transverse momenta. More details can be found in other talks in this volume[7] or in [3] and [6]. In this context the "Lipatov equation" is just the (lowest order form of the) Schwinger-Dyson equation for two-reggeon exchange. A major point that we want to emphasized very strongly is that the complete structure of the reggeon diagrams obtained is the simplest allowed within the general formalism of "Analytic Multi-Regge Theory" [3]. It is this feature which allows us to confidently extrapolate the leading and next-toleading results to very complicated multiparticle /multi-Regge amplitudes.

\section{THE TRANSVERSE MOMENTUM CUT-OFF}

Since the reggeon diagram formalism of massive gauge theories is very well understood in terms of general multi-Regge theory, we would clearly like to use it to study the Regge behavior of hadrons and the soft Pomeron in massless gauge theories. There are a number of general arguments, including those listed below, which imply that as we take the massless limit we should keep only the low $k_{\perp}$ part of the diagrams.

i) The dispersion theory construction is, as we have discussed, dominated by the "on-shell" region of Feynman diagrams and as a result higherorder reggeon diagrams are only well-defined close to such regions $[6,7]$.

ii) Regge trajectory functions have the general form illustrated below and, as is shown, fall-off at large $t$

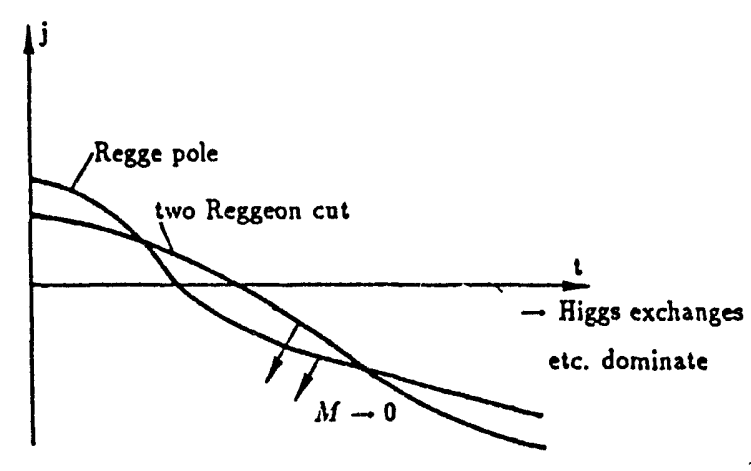

Because of this fall-off, the non-reggeized, nonasymptotically free Higgs scalar contributions to production amplitudes (which we would like to see decouple in the massless limit) will dominate over multi-Regge exchanges at sufficiently large $t$. As is also illustrated, this problem intensifies as the gluon mass $M \rightarrow 0$.

iii) The last feature is related to the perturbative property that the scalar "Higgs Sector", used to give the gluons their mass, decouples smoothly from individual Feynman diagrams only when an ultra-violet cut-off is present.

iv) The lattice gauge theory principle of complimentarity[8], that we use to argue that there is no Higgs/confinement phase transition when fundamental representation scalars are used, also requires an ultra-violet cut-off. 
v) There is a deeper issue that the infrared (or "infinite-volume") limit, which is non-trivial because of the presence of topological gauge fields, should be taken before the ultra-violet (or "continuum") limit is taken utilizing asymptotic freedom.

For the major part of our analysis, therefore, we keep only the $\left|k_{\perp}\right|<\lambda_{\perp}$ part of reggeon diagrams (where $\lambda_{\perp}$ is a fixed cut-off). Note that this is the part of the Lipatov equation that a strictly perturbative theorist would like to subtract[9] into the "non-perturbative" part of parton distributions.

\section{INFRARED BEHAVIOR OF REGGEON DIAGRAMS}

If we now consider the massless limit of a pure gluon theory then the "on-shell" dispersion theory approach that we have outlined above immediately implies that there can not be transverse momentum infrared divergences associated with on-shell gluons in the reggeon intermediate states. An on-shell massless gluon can not decay into two massless gluons because of helicity conservation. As a result a general reggeon interaction satisfies a "Ward Identity property" which we can derive as follows

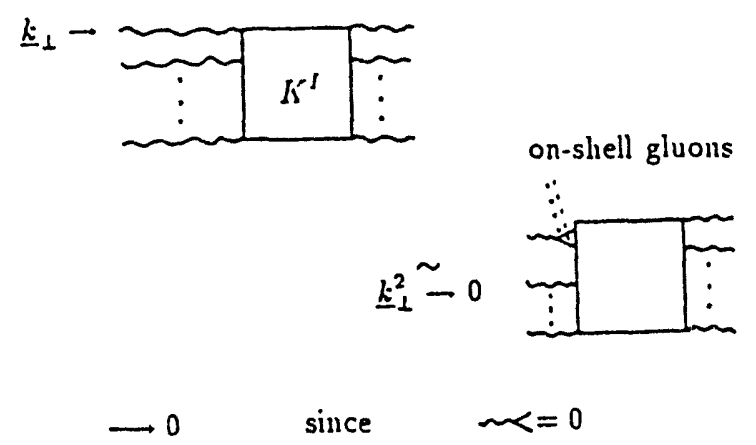

We refer to this as a Ward Identity property because it is normally derived for Feynman diagrams by conventional Ward Identity manipulations. In such manipulations the usual concern is with ultraviolet divergences. Here we are asking whether the infrared mass-shell region, which effectively controls the Regge limit, causes any problem. The answer is that in a pure gluon theory it does not. The resulting vanishing of reggeon interactions at small $k_{\perp}^{2}$ is sufficient to ensure that there are no infrared divergences resulting from integration over the transverse momenta of a reggeon state (provided the the state has overall non-zero transverse momentum). Therefore divergences can occur only within the reggeon interactions $K^{I}$ (that is the Lipatov kernels).

Within the $K^{I}$ there are divergences, for example, of the form

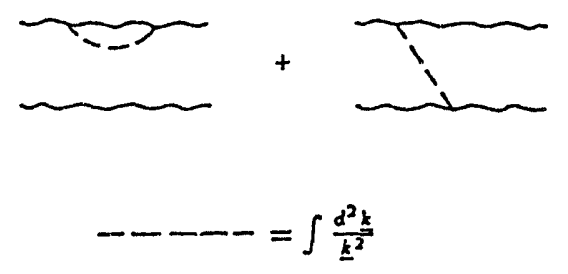

As we have indicated, a distinct $t$-channel color $I$ can be assigned to each reggeon interaction. For $\mathrm{SU}(2)$ gauge theory in particular the interactions carry "isospin" $I=0,1, \cdots$. All interactions that carry non-zero color do indeed contain divergences, and the iteration of such interactions results in the vanishing of all reggeon diagrams with non-zero color as $M \rightarrow 0$. (Since the iteration of divergences is an exponentiation in rapidity space, this effect could be regarded as a generalized Sudakov suppression).

The divergences zancel, however, within all color zero interactions. That is such interactions are both infrared finite and satisfy the Ward Identity property described above. Consequently all color zero reggeon states survive the $M \rightarrow 0$ limit. (This result includes the infrared finiteness properties of the Lipatov equation.) This produces two related, and from our point of view equally unwanted, phenomena.

i) Although the surviving diagrams are finite, there is non-analytic behavior at $q^{2}=0$. Therefore, such diagrams can not represent the Regge limit of a confining theory with no massless particles.

ii) The Ward Identity "softening" of reggeon interactions at $k_{\perp}^{2}=0$ leads to the dominance of large $k_{\perp}$ and (if the $k_{\perp}$ cut-off is removed) the 
emergence of the Lipatov Pomeron[1]. When the Lipatov equation is applied to the evolution of small $x$ parton distributions this is, essentially, what produces the parton saturation phenomenon[2].

Since the decoupling of gluon reggeon interactions on-shell is so closely related to the Ward Identities preserving gauge-invariance and to the zero mass of the gluon, it would appear to be very difficult to avoid these phenomena! Massless quarks can change this situation, however.

\section{MASSLESS QUARKS AND CONFINEMENT}

Because a massless on-shell gluon can decay into a pair of on-shell massless quarks, it is clear that the above argument for the vanishing of reggeon interactions at $k_{\perp}^{2}=0$ does not go through in the presence of massless quark loops. In general, of course, the on-shell regions of quark loops are not sufficiently singular to produce any violation of Ward Identity arguments. However, because of the mass-shell dominance of the buildup of Regge behavior described above, the Regge limit enhances the contribution of on-shell regions. We shall now argue that, as a consequence, the infrared violation of "Ward Identity Properties" by massless, Regge region, quarks provides the mechanism that produces confinement in reggeon diagrams. The phenomena we discuss are related, at least indirectly, to the recently discoveredi10] infrared "anomaly" in the crosssection for the production of a massless electron/ positron pair by a longitudinal photon in massless QED.

In general it is not easy to isolate quark reggeons within gluon reggeon diagrams because gluon exchanges dominate quark exchanges by powers of the energy involved. However, in a non-abelian theory a reggeon diagram involving a reggeized quark loop can be isolated[3] (within a multiparticle amplitude) by requiring particular quark quantum number exchange. By taking the appropriate multiple discontinuity (indicated by dashed lines) we arrive at a transverse momentum interaction as follows

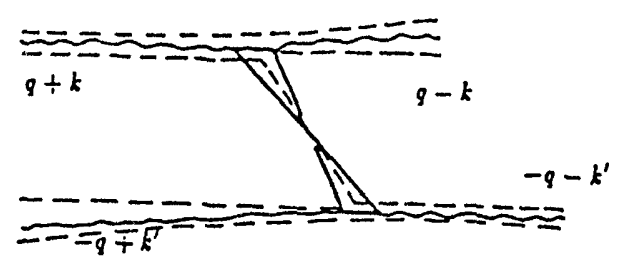

reggeon diagram

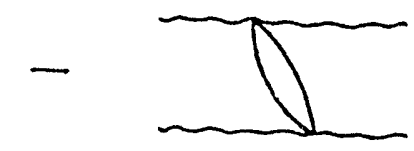

transverse momentum

interaction in gluon

reggeon diagrams

The sum of such diagrams produces a vital contribution to the $I=0$ four reggeon interaction (or Lipatov kernel) having the form (which we give explicitly to illustrate the point)

$$
\begin{aligned}
\int d^{2} p & \frac{\operatorname{Tr}\left[(p-q) k(p+\phi) k^{\prime}\right]}{(p+q)^{2}(p-q)^{2}} \\
& \rightarrow 0 \cdot 8 \pi\left[\frac{2(q \cdot k)\left(\dot{q} \cdot k^{\prime}\right)}{q^{2}}-k \cdot k^{\prime}\right] \\
q & \rightarrow \overrightarrow{ \pm k}^{\prime} \pm k^{\prime} 8 \pi k \cdot k^{\prime}
\end{aligned}
$$

showing that the quark loop does indeed produce a reggeon interaction which does not vanish at zero transverse momentum.

This last result generalizes in that if we consider an arbitrary $(I=0)$ reggeon state and consider a limit in which some subset of transverse momenta vanish, there are again quark-loop multireggeon interactions which do not vanish in this limit. That is
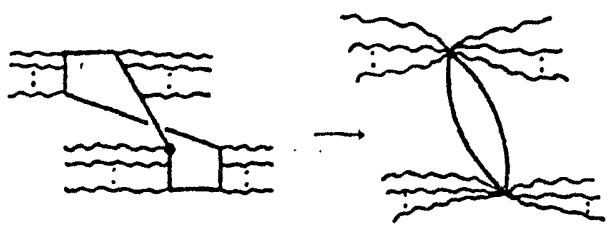
It is crucial that the reggeon amplitudes which violate Ward Identity properties in this manner are always defined by multiple discontinuities which determine[3] that they are not related to particle scattering amplitudes. This is a very technical point which we will not elaborate on here. However, it is of vital importance because it guarantees that there is no violation of the reggeization properties which are, presumably, the most direct expression of the underlying gauge invariance of the theory. Thus while the quark loop interactions do not violate gauge invariance they do result in infrared divergences associated directly with the $I=0$ reggeon states which caused the "unwanted phenomena" described above. Reggeon unitarity then prescribes that such divergences are iterated, with the result that (almost) all gluon reggeon states are removed, implying perhaps that confinement is indeed emerging from the formalism.

\section{ANOMALOUS INTERACTIONS}

An $I=0$ multigluon reggeon state can avoid the suppression by massless quark interactions that we have just described only if each gluon in the reggeon state carries $k_{\perp}=0$. To allow this, such a gluon configuration must accompany an $I=0$ infrared finite state which carries the total transverse momentum. For $\mathrm{SU}(2)$ gauge theory this must be a quark state. To also avoid any interaction with this quark state which would produce further suppression via divergences, the gluon state must carry "anomalous quantum numbers". For SU(2) gauge theory, the only possibility is an "anomalous odderon" configuration, that is a set of gluons with $I=0$, color parity $C=$ +1 and signature $\tau=-1$ (c.f. $\epsilon_{i j k} A^{i} A^{j} A^{k}$ ).

A key role is now played[3] by a triangle anomaly reggeon diagram, that is an on-shell quark transverse momentum triangle diagram. It can be shown[3] that this diagram contributes only in very special "triple Regge" kinematics. However, it then provides a crucial coupling for the anomalous odderon via an infrared divergence violating helicity conservation. That is

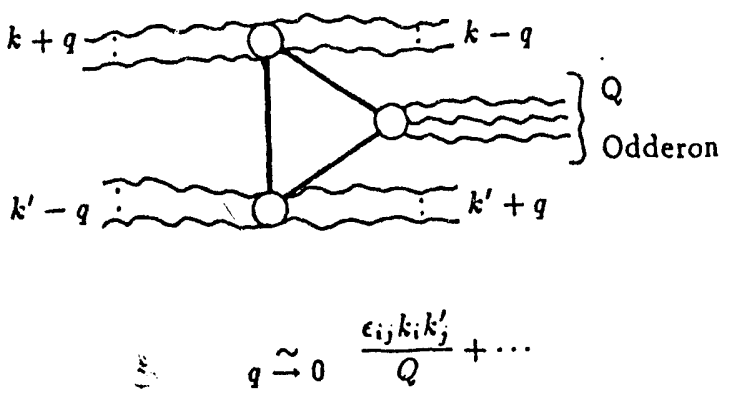

The singular $Q$ - dependence is what produces an odderon coupling violating helicity conservation. To extract the full significance of the resulting structure of couplings and divergences requires extensive use of the machinery of Multi-Regge theory [3] and here we will just briefly summarize what we believe is the outcome.

In a multi-Regge amplitude, there is an overall infrared divergence for each surviving reggeon state. This divergence has to be factorized off to define a reggeon condensate - with the quantum numbers of the anomalous odderon. For SU(2) gauge theory there is no Pomeron, and the only physical reggeon states are mesons formed from quark/antiquark reggeons in the reggeon condensate background. The anomalous odderon gluon state producing the condensate is closely related to the "Winding Number Operator"

$$
K^{\circ}=-\frac{2}{3} \epsilon_{o \mu \nu \delta} \epsilon^{i j k} A_{\mu}^{i} A_{\nu}^{j} A_{\delta}^{k}+\epsilon_{o \mu \nu \delta} F_{\mu \nu}^{i} A_{\delta}^{i}
$$

i)Consequently, there is a close parallel[3] between the reggeon condensate origin of confinement in our formalism and the solution[11] of the twodimensional massless Schwinger model. In both cases it is the role of the anomaly when massless fermions are present which dominates the dynamics.

At this point we should emphasize that the reggeon diagram infrared analysis does not include effects associated with the growth of the gauge coupling. As a result the dynamical results we describe are immediately applicable only when there is a sufficient number[12] of massless quarks to prevent the infrared growth of the cou- 
pling (and also there is a transverse momentum cut-off).

\section{THE QCD SOFT POMERON}

The analysis we have outlined can be extended to discuss the Pomeron in a general SU(N) gauge theory by utilizing a staged restoration of the gauge symmetry via the decoupling of fundamental representation Higgs scalars. It produces an attractive picture of Pomeron trajectories in higher gauge groups[3] which is closely related to properties of transverse loops (Wilson loops) of color flux.

We study the Pomeron in QCD by first constructing the theory in which the $\mathrm{SU}(3)$ gauge symmetry is broken to $\mathrm{SU}(2)$. In this case the Pomeron appears as an $\mathrm{SU}(2)$ singlet reggeized gluon in the background of an SU(2) reggeon condensate (which switches the signature). Quark masses (at least the light quark masses) can be smoothly added after the infrared limit giving $\mathrm{SU}(2)$ gauge symmetry is taken. The physical QCD (soft) Pomeron is then obtained by restoring the full $\mathrm{SU}(3)$ gauge symmetry. There are many attractive features to the construction,

i) the explicit utilization of reggeon diagrams makes it straightforward to organise all contributions into a Pomeron Reggeon Field Theory we find that the theory is in the "Super-Critical" phase[3] as long as the gauge symmetry remains broken to $\mathrm{SU}(2)$,

ii) the Pomeron is truly dominated by low transverse momenta and satisfies reggeon unitarity (and perhaps even a multiperipheral bootstràp!),

iii) parton-model concepts are effectively extended down to low transverse momentum - the soft Pomeron can be thought of as single (reggeized) gluon exchange plus a colored rearrangement of the fermion sea in scattering hadrons. This gives a basis for the phenomenological success of the "Pomeron-photon analogy" and the additive quark model[13].

Using Reggeon Field Theory we can argue that as $S U(3)$ gauge symmetry is restored and the cut-off $\lambda_{\perp}$ is raised, there exists a critical value $\lambda_{\perp}=\lambda_{c}$ at which the Pomeron becomes Critical $[14]\left(\alpha_{\mathrm{B}}(0)=1\right) . \lambda_{c}$ depends on the number of flavors $N_{j}$ and $\lambda_{c}=\infty$ only for $N_{\jmath}=16$, or if appropriate higher color quarks are present[3]. Note that as the Pomeron becomes Critical the condensate automatically disappears and all gluons that comprise the Pomeron share the available transverse momentum.

\section{SMALL X PARTON DISTRIBUTIONS}

Clearly the inclusion in the Lipatov equation of the massless-quark interactions we have described has a drastic effect - there are infrared divergences. A major question for the perturbative derivation of "Hard Pomeron" modifications[2] of small $x$ parton distributions is whether a lower transverse momentum cut-off (that is $k_{\perp}>\lambda_{\perp}$ is imposed) which excludes the true Regge region, justifies neglect of these interactions? Perhaps not for any fixed $\lambda_{\perp}$ ! Another question is whether finite quark masses can be used to restrain the effect, or to determine the appropriate transverse cut-off?

It seems inevitable that the solution of the parton saturation probiem is deeply related to the origin of true Regge behavior for the Pomeron, that is the origin of low transverse momentum dominance. As I have indicated, I believe the light quarks, the anomaly of the fermion sea etc. are behind this. My suspicion is that parton saturation should not survive the inclusion of these effects. Clearly there is much to do to bring the $k_{\perp}<\lambda_{\perp}$ analysis that I have outlined to a calculational level where it would begin to compare with standard perturbative QCD applied to $k_{\perp}>\lambda_{\perp}$. However, if I push my present understanding[3] of the relationship between the QCD "soft Pomeron" and Reggeon Field Theory as far as possible, the following $\lambda_{\perp}$ dependence emerges.

As we have already implied, given the number of quark flavors and their masses, a critical value $\lambda_{c}$ of the cut-off $\lambda_{\perp}$ is determined. If we then consider calculating the soft Pomeron from $Q C D$ with a varying value of $\lambda_{\perp}$ we will find

i) $\lambda_{\perp} \ll \lambda_{c}$

The Pomeron is Super-Critical, or equivalently , 
the Pomeron is a reggeized gluon (in a condensate background). This leads to the Pomeron-photon analogy, the additive quark model result for total cross-sections, etc.

ii) $\lambda_{\perp}=\lambda_{c}$

The Pomeron is Critical, implying that we can calculate the diffraction peak, and many other diffractive amplitudes, in detail. It would be ideal if $k_{\perp}>\lambda_{c}$ were also calculable with reasonable accuracy within perturbative QCD.

iii) $\lambda \gg \lambda_{c}$

The Pomeron is Sub-Critical, implying that "bare Pomeron" RFT should be used. Recall that a bare Pomeron pole gives $\sigma_{T} \sim s^{\alpha_{0}-1}$ with $\alpha_{0}>1$ and it follows from the RFT formalism[15] that increasing $\lambda_{\perp}$ decreases $\alpha_{0}$.

iv) $k_{\perp}>\lambda_{\perp}$

For sufficiently large $\lambda_{\perp}$, the RFT formalism can not be used and (presumably) perturbative QCD should be used, giving the Lipatov Pomeron[1] etc. But the Lipatov Pomeron gives $\sigma_{T} \sim s^{\alpha_{0}-1}$ where[16] $\alpha_{0}$ again decreases as $\lambda_{\perp}$ increases.

Clearly iii) and iv) are compatible with the idea that there is no physical significance to $\lambda_{\perp}$. However, these properties also suggest that in practise it may be difficult to make a phenomenological distinction between "Hard" and "Soft" Pomeron effects. This could clearly make elusive any experimental search for the Lipatov Pomeron as a well-defined "semi-hard" concept.

\section{REFERENCES}

[1] L.N. Lipatov, in "Perturbative QCD" edited by A.H. Mueller, World Scientific (1989).

[2] For example: L. V. Gribov, E. M. Levin and M. G. Ryskin, Phys. Rpts. 100C, 1 (1983); A. H. Mueller and H. Navelet, Nucl. Phys. B282, 727 (1987); R. K. Ellis and D. A. Ross, Nucl. Phys. B345, 79 (1990),

[3] A. R. White, Int. J. Mod. Phys. A11, 1859 (1991) and "Analytic Multi-Regge Theory and the Pomeron in QCD : II. Gauge Theory Analysis" ANL preprint - to appear, see also Nucl. Phys. B (Proc. Suppl.) 12, 190, (1990) and 25B, 167 (1992).

[4] E. A. Kuraev, L. N. Lipatov and V. S. Fadin, Sov. Phys. JETP 44, 443 (1976) and 45, 199 (1977);

[5] H. Cheng and C. Y. Lo, Phys. Rev. D13, 1131 (1976) and D15, 2959 (1977).

[6] J. Bartels, Nucl. Phys. B151, 293 (1979), B175, 365 (1980) and DESY Preprint DESY 91-074 (1991)

[7] See the presentation by J. Bartels in particular.

[8] E. Fradkin and S. H. Shenker, Phys. Rev. D19, 3682 (1979); T. Banks and E. Rabinovici, Nucl. Phys. B160, 349 (1979).

[9] J. C. Collins and R. K. Ellis, Nucl. Phys B (Proc. Suppl.) 18C, 80 (1991).

[10] A. S. Gorsky, B. L. Ioffe and A. Yu. Khodjamirian, Phys. Letts. B227, 474 (1989). I am grateful to $G$. Sterman for pointing out the possibility of this relationship.

[11] N. S. Manton, Ann. Phys: 159, 220 (1985).

[12] D. J. Gross and F. Wilczek, Phys. Rev. D8, 3633 (1973).

[13] P. V. Landshoff, Nucl. Phys. B (Proc. Suppl.) 12, 397 (1990).

[14] A. A. Migdal, A. M. Polyakov and K. A. TerMartirosyan, Zh. Eksp. Teor. Fiz. 67, 81 (1974); H. D. I. Abarbanel and J. B. Bronzan, Phys. Rev. D9, 2397 (1974).

[15] R. L. Sugar and A. R. White, Phys. Rev. D1?, 4074 (1974).

[16] A. D. Martin, these proceedings. 

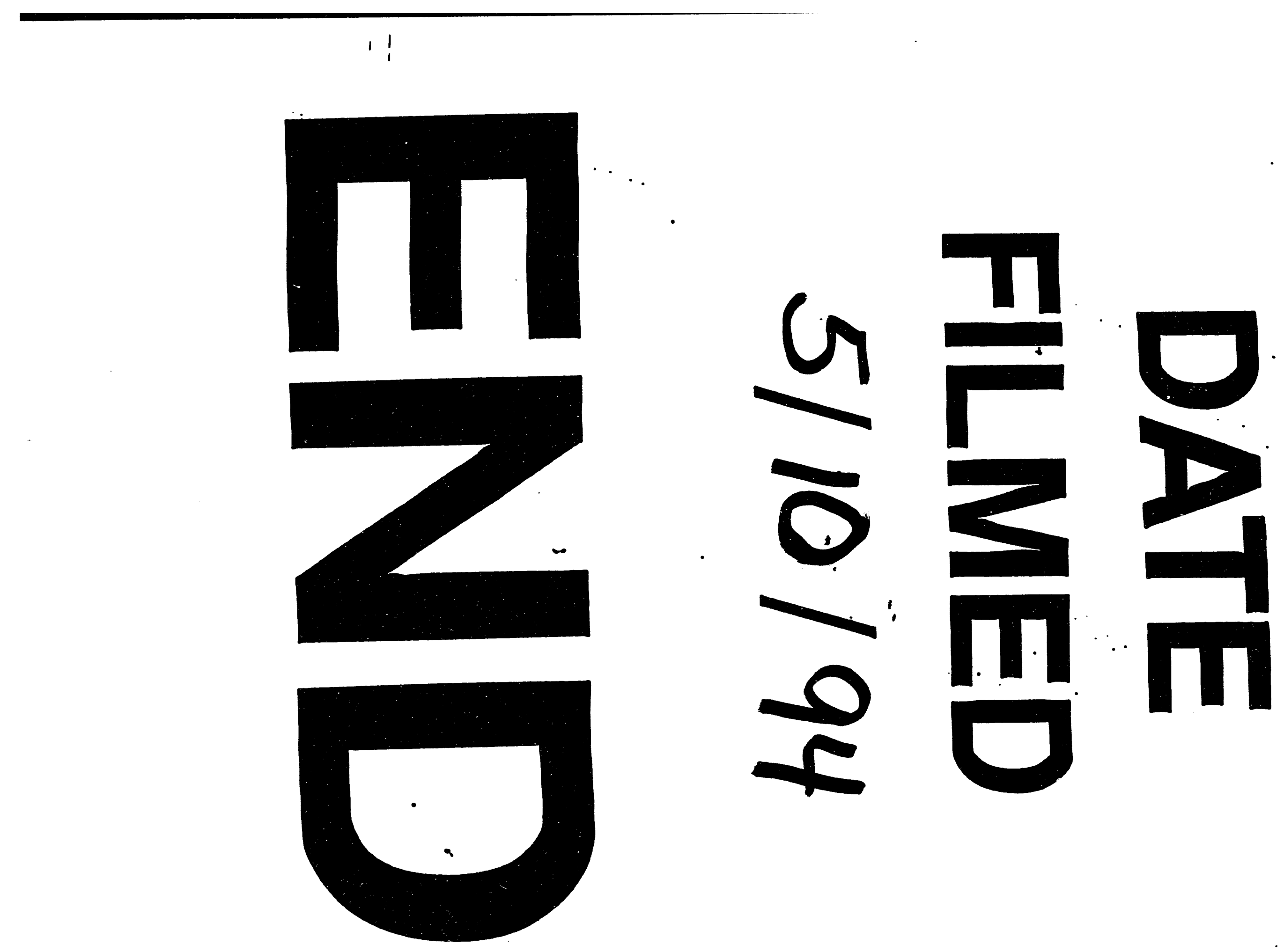
